\title{
DIAGNÓSTICO DIFERENCIAL ENTRE TRANSTORNO DO ESPECTRO AUTISTA (TEA) E DISTÚRBIO ESPECÍFICO DE LINGUAGEM (DEL)
}

\author{
DIFFERENTIAL DIAGNOSIS IN AUTISM SPECTRUM DISORDER (ASD) AND \\ SPECIFIC LANGUAGE IMPAIRMENT (SLI)
}

\section{Liz Passos Nascimento Souza ${ }^{1}$}

RESUMO: O Transtorno do Espectro Autista é caracterizado principalmente por prejuízos na comunicação e interação social e padrões restritos e repetitivos de comportamento, atividades e interesses. O Distúrbio Específico de Linguagem apresenta prejuízos na linguagem, o portador deste distúrbio apresenta dificuldade para compreender ou produzir palavras e/ou frases em uma conversa. Em alguns casos, tentam compensar o prejuízo para usar palavras com gestos e outras expressões corporais. Ambos são transtornos do neurodesenvolvimento, apresentam características linguísticas semelhantes em seus sintomas e diferenciá-los torna-se fundamental para a condução do tratamento adequado para cada um deles, uma vez que sua não identificação pode atrasar o tratamento, acarretando prejuízo pessoal, social e profissional aos portadores. Este trabalho tem como objetivo investigar as diferenças e traçar uma linha de raciocínio para o diagnóstico diferencial de TEA e DEL. Foi realizada uma pesquisa exploratória através de revisão bibliográfica e nota-se que a grande diferença entre eles é a debilidade funcional, onde os portadores de TEA apresentam maior comprometimento, bem como prejuízos nas áreas de cognição e interação social, diferenciando-se dos portadores de DEL.

Palavras-chave: Transtorno do Espectro Autista. Distúrbio Específico de Linguagem. Linguagem. Interação Social.

ABSTRACT: Autism Spectrum Disorder is mainly characterized by impairments in communication, social interection and restricted and repetitive patterns of behavior activities and interests. The Specificic Language Impairment has language impairment, the person with this disorder has difficulty understanding or producing words and/or phrases

\footnotetext{
I Psicóloga Clínica Cognitivo-Comportamental. Possui graduação em Psicologia e especialização na área de Avaliação Neuropsicológica pela Pontifícia Universidade Católica do Rio de Janeiro. Atuou na área de saúde mental, tendo estagiado no Hospital de Custódia e Tratamento Psiquiátrico Roberto de Medeiros. Atualmente dedica-se ao atendimento clínico em terapia cognitivo-comportamental,e avaliação e reabilitação neuropsicológica de crianças, adolescentes, adultos e idosos. Atuação na área de Terapia CognitivoComportamental, terapia comportamental e Neuropsicologia em transtornos de neurodesenvolvimento e dificuldades de aprendizagem.
} 
in a conversation. In some cases, They try to make up for the harm by using words with gestures and other bodily expressions. Both are neurodevelpmental disorders, have similar linguistic characteristics in their symptoms and differentiating them is essencial for conducting the appropriate treatment for each of them, since their non-identification can delay the treatment, resulting in personal, social and professional service to carriers. This work aims to investigate the diferences and trace a line of reasoning for the differential diagnosis of ASD and SLI. An exploratory research was carried out through a literature review and it is noted that the big difference bitween them is functional weakness, where ASD patients have greater impairment, as well as losses in the areas of cognition and social interaction, differing from those with SLI.

Keywords: Autism Spectrum Disorder. Specific Language Impairment. Language. Social interaction.

\section{INTRODUÇÃO}

$\mathrm{Na}$ última década, houve um grande avanço no que tange a conscientização do transtorno do espectro autista, com isso, obteve-se maior reconhecimento do transtorno e maior número de pesquisas com foco na causa e intervenções com crianças pequenas (Simms and Jin, 2015).

O transtorno do espectro autista (TEA), e o distúrbio específico de linguagem (DEL) são transtornos do neurodesenvolvimento, seus sintomas podem ser detectados desde a primeira infância, e manifestam-se de forma semelhante e não especifica, ou seja, não há apenas um padrão de manifestação, o que torna seu diagnóstico desafiador (Faé et al., 2018). Ambos apresentam características semelhantes no campo da linguagem e interação social, o que justifica o fator confusional entre eles.

O objetivo do presente estudo é discorrer sobre os possíveis critérios de diagnósticos diferenciais entre TEA e DEL, bem como instrumentos sensíveis nessa identificação. A importância da identificação precoce dos dois transtornos é fundamental, pois uma vez não identificado, acarreta atraso no tratamento, prejuízo pessoal e profissional ao paciente (Faé et al., 2018). Os dois transtornos, embora sejam transtornos do neurodesenvolvimento e assemelhem em muitos fatores, possuem indicações de tratamentos e intervenções completamente diferentes, então para maior eficácia de melhora, faz-se necessário novamente, o diagnóstico diferencial. 


\section{Conceituando TEA e DEL}

\section{I Transtorno do Espectro Autista}

O transtorno do espectro autista (TEA) é um transtorno do neurodesenvolvimento que surge normalmente nos primeiros três anos de vida da criança.

Durante os anos 40, foi iniciada uma serie de discussões acerca do que hoje conhecemos como "autismo", quando o psiquiatra austríaco Leo Kanner (1943) trouxe a definição do termo. Ele utilizou autismo para definir indivíduos com a capacidade de se relacionar com outras pessoas "de uma maneira comum", gravemente prejudicada, preferindo ficarem sozinhos reclusos em seus próprios mundos. Esses indivíduos também apresentavam comunicação e interação social afetadas, com dificuldade em usar a linguagem para transmitir significado aos outros. Kanner observou ainda, que atividades espontâneas eram limitadas bem como a variedade de seus comportamentos.

Kanner (1943) ao descrever o conceito de autismo ressaltou a impossibilidade de comunicação e linguagem. Ele afirma que autistas não possuem linguagem e que suas expressões são ecolalia (repetição de frases/palavras). Três anos depois, relata que não é possível afirmar que exista de fato uma ausência de linguagem levando em consideração a capacidade criadora desses indivíduos, mas que as construções de linguagem só fazem sentido em um contexto.

Esses apontamentos iniciais serviram de base para o diagnóstico do que chamamos hoje de transtorno do espectro autista (TEA), e de outros transtornos do desenvolvimento.

O DSM-5 (AMERICAN PSYCHIATRIC ASSOCIATION, 2014) forneceu uma definição atualizada de TEA que destacou a gravidade de sintomas em duas esferas principais: social (comunicação e interação social) e não social (padrões restritos e repetitivos de comportamentos, interesses ou atividades). Esse novo enquadramento, possibilitou a distinção de indivíduos com falta de interesse social primária (TEA), dos que possuem dificuldades na interação social, devido a prejuízos nas habilidades comunicativas.

Crianças dentro do TEA podem ignorar colegas, pais ou cuidadores e não identificar sentimentos dos outros (quando estão aflitos ou precisam de ajuda). Segundo Simms e Jin (2015), em muitas crianças a adesão inflexível a rotina e rituais muito específicos não são 
funcionais e existe uma resistência marcada a qualquer tipo de mudança nesses padrões, favorecendo seu isolamento no ambiente ao redor devido a insistência em aderir regras e rigidez do pensamento.

Os padrões restritos e repetitivos de comportamento também são características importantes de definição do TEA. Tais comportamentos incluem estereotipias motoras simples (abanar as mãos ou estalar dos dedos), uso repetitivo de objetos (girar moedas, enfileirar objetos), e fala repetitiva (ecolalia, uso estereotipado de palavras).

Muitos indivíduos dentro do TEA possuem déficits de linguagem, variando de ausência total da fala, passando por atrasos de linguagem, compreensão reduzida da fala e ecolalia. Mesmo com habilidades linguísticas formais intactas (vocabulário, gramática), o DSM-5 (AMERICAN PSYCHIATRIC ASSOCIATION, 2014) aponta o uso da linguagem enquanto comunicação social recíproca prejudicada dentro do TEA, que costuma ser unilateral, sem reciprocidade, usada mais para solicitar, rotular do que de fato comentar, compartilhar sentimentos e/ou conversar. São apresentados déficits na reciprocidade social evidente em crianças pequenas no transtorno que podem apresentar pequena ou nenhuma capacidade de iniciar interações sociais e compartilhar emoções, além de imitação reduzida ou ausente.

Atualmente, o DSM-5 classifica o TEA em 3 níveis diferentes. No Nível I: "exigindo apoio", ausência de apoio os prejuízos na comunicação social são perceptíveis. Estão presentes dificuldades para iniciar interações sociais com interesse reduzido. Por exemplo, um indivíduo que consegue falar frases completas e se envolver na comunicação, porém apresenta falhas de conversação com o outro e as tentativas de amizade são estranhas e geralmente falhas. No aspecto comportamental, nota-se comportamento inflexível com interferência no funcionamento em um ou mais contextos. Dificuldades na troca de atividades, problemas na organização e planejamento são desafios para a independência.

O Nível 2: "exigindo apoio substancial", estão presentes prejuízos graves na comunicação social verbal e não verbal, prejuízos sociais também são perceptíveis ainda que na presença de apoio, limitações para início nas interações sociais, e resposta reduzida ou anormal para aberturas sociais partindo do outro. Exemplo, um indivíduo que fala frases 
simples, mas sua interação se limita a interesses especiais reduzidos e comunicação não verbal significativamente estranha. Apresenta inflexibilidade do comportamento, dificuldade para lidar com a mudança ou outros comportamentos restritos/repetitivos aparecem de maneira suficiente para serem óbvios a um observador casual e interferem no funcionamento de diversos contextos. Sofrimento e/ou dificuldade de mudar o foco ou as ações.

O Nível 3: "exigindo apoio muito substancial", apresentam-se déficits graves na comunicação social verbal e não verbal, prejuízos no funcionamento, limitação em iniciar interações sociais e resposta mínima a aberturas sociais propostas pelo outro. Por exemplo, uma pessoa que fala inteligível com poucas palavras, que raramente inicia interações, e quando o faz, tem abordagens incomuns apenas para satisfazer a necessidades e reagem apenas a abordagens sociais muito diretas. Apresenta comportamento inflexível, extrema dificuldade a lidar com mudança ou outros comportamentos restritos/repetitivos interferem acentuadamente no funcionamento em todos os âmbitos. Grande sofrimento/dificuldade para mudar foco ou ações.

\subsection{Critérios Diagnósticos para Transtorno do espectro autista (TEA) - Definição DSM-5}

A. Déficits persistentes na comunicação social e interação social através de múltiplos contextos, como manifestado a seguir, atualmente ou por história (exemplos são ilustrativos, não exaustivos):

i. Déficits na reciprocidade socioemocional, variando, por exemplo, de abordagem social anormal e dificuldade para estabelecer uma conversa normal a compartilhamento reduzido de interesses, emoções ou afeto, a dificuldade para iniciar ou responder a interações sociais.

ii. Déficits nos comportamentos comunicativos não verbais usados para interação social, variando, por exemplo, de comunicação verbal e não verbal pouco integrada a anormalidade no contato visual e linguagem corporal ou déficits na compreensão e uso gestos, a ausência total de expressões faciais e comunicação não verbal.

iii. Déficits para desenvolver, manter e compreender relacionamentos, variando, por exemplo, de dificuldade em ajustar o comportamento para se adequar a 
contextos sociais diversos a dificuldade em compartilhar brincadeiras imaginativas ou em fazer amigos, a ausência de interesse por pares.

iv. Especificar a gravidade atual: A gravidade baseia-se em prejuízos na comunicação social e em padrões de comportamento restritos e repetitivos

B. Padrões restritos e repetitivos de comportamento, interesses ou atividades, conforme manifestado por pelo menos dois dos seguintes, atualmente ou por história prévia (os exemplos são apenas ilustrativos, e não exaustivos).

i. Movimentos motores, uso de objetos ou fala estereotipados ou repetitivos (p. ex., estereotipias motoras simples, alinhar brinquedos ou girar objetos, ecolalia, frases idiossincráticas).

ii. Insistência nas mesmas coisas, adesão inflexível a rotinas ou padrões ritualizados de comportamento verbal ou não verbal (p. ex., sofrimento extremo em relação a pequenas mudanças, dificuldades com transições, padrões rígidos de pensamento, rituais de saudação, necessidade de fazer o mesmo caminho ou ingerir os mesmos alimentos diariamente).

iii. Interesses fixos e altamente restritos que são anormais em intensidade ou foco (p. ex., forte apego a ou preocupação com objetos incomuns, interesses excessivamente circunscritos ou perseverativos).

iv. Especificar a gravidade atual: A gravidade baseia-se em prejuízos na comunicação social e em padrões restritos ou repetitivos de comportamento

C. Os sintomas devem estar presentes precocemente no período do desenvolvimento (mas podem não se tornar plenamente manifestos até que as demandas sociais excedam as capacidades limitadas ou podem ser mascarados por estratégias aprendidas mais tarde na vida).

D. Os sintomas causam prejuízo clinicamente significativo no funcionamento social, profissional ou em outras áreas importantes da vida do indivíduo no presente.

E. Essas perturbações não são mais bem explicadas por deficiência intelectual (transtorno do desenvolvimento intelectual) ou por atraso global do desenvolvimento. Deficiência intelectual ou transtorno do espectro autista costumam ser comórbidos; para fazer o diagnóstico da comorbidade de transtorno do espectro autista e deficiência intelectual, a comunicação social deve estar abaixo do esperado para o nível geral do desenvolvimento. 
F. Na Classificação dos Transtornos Mentais e de Comportamento (CID-ıo), o Transtorno do Espectro Autista está classificado dentro do Transtorno Invasivo do desenvolvimento. Existem 8 classificações dentro do dos Transtornos invasivos do desenvolvimento: (I) Autismo infantil; (2) Autismo atípico; (3) Síndrome de Rett; (4) Outro transtorno desintegrativo da infância; (5) Transtorno de hiperatividade associado a retardo mental e movimentos estereotipados; (6) Síndrome de Asperger; (7) Outros transtornos invasivos do desenvolvimento; (8) Transtorno invasivo do desenvolvimento, não especificado.

\subsection{Distúrbio específico de linguagem (DEL)}

A linguagem oferece a possibilidade de comunicação entre pessoas. Entra também como meio pelo qual se dão processos de interações de aprendizagem e sociais. De acordo com Simms e Jin (2015), as habilidades de comunicação abrangem todas as ações e habilidades que envolvem troca de informação, pensamentos e sentimentos entre pessoas. Assim, a habilidade de comunicação tem componentes verbais e não verbais. Elas se referem ao uso e compreensão de palavras e frases. Os componentes essenciais da linguagem incluem produção de sons (fonemas), significado de palavras (semântica), gramática (sintaxe) e ritmo e entonação da fala (prosódia). As habilidades linguísticas de ordem superior englobam o uso funcional e apropriado das habilidades verbais e não verbais para uma comunicação eficaz (pragmática).

Um distúrbio do desenvolvimento da linguagem não devido à deficiência intelectual ou física é referido na literatura como um distúrbio de linguagem específico (DEL), ou como o DSM-5 (AMERICAN PSYCHIATRIC ASSOCIATION, 2014) refere: Transtorno da Linguagem.

De acordo com Crestani et al. (2013), o distúrbio específico de linguagem (DEL) é caracterizado por importantes prejuízos na linguagem, tais como atrasos ou alterações na aquisição, pode afetar a fala, escuta e leitura de uma criança. Ele apresenta variabilidade em suas manifestações, variando também o nível de gravidade e mutação ao longo do desenvolvimento.

A criança com DEL possui dificuldade em compreender ou produzir palavras e frases durante uma conversa. Segundo Faé et al, (2018) pode de apresentar alterações em habilidades de linguagem oral, receptiva que é a capacidade de receber e compreender o que 
o outro está falando, expressiva que é a capacidade de produzir fala de forma a ser compreendida verbalmente por outro individuo, ou ambas.

Em alguns casos, Simms e Jin (2015) afirmam que as crianças com DEL tentam compensar a incapacidade de usar palavras, utilizando gestos e outras expressões corporais, mas frustração e birras são comuns. Embora muitos pré-escolares utilizem rotinas e horários para ajudar a organizar atividades, aqueles com deficiências na linguagem podem ter uma necessidade maior de rotina, apresentando mais resistência em situações novas ou desconhecidas. Quando as crianças com DEL começam a usar palavras, elas podem repetir frases ou diálogos de filmes ou histórias de uma maneira ecolálica.

O DSM-5 (AMERICAN PSYCHIATRIC ASSOCIATION, 2014) coloca dois critérios principais para diagnosticar o transtorno de linguagem: (I) dificuldades persistentes na aquisição e uso da linguagem em suas variadas modalidades (falada, escrita, sinais ou outra) devido a prejuízos na compreensão ou produção; (2) capacidades linguísticas estão abaixo do esperado para idade, limitando a comunicação efetiva, participação social, sucesso acadêmico e/ou desemprenho profissional; além de exclusão por outros atrasos, por isso o termo "específico".

O DEL costuma afetar o vocabulário e gramática, o que consequentemente limita a capacidade de discurso. As primeiras palavras e expressões da criança, possivelmente surgem com atraso, o vocabulário é menor e menos variado do que o esperado, as frases são mais curtas e menos complexas. Estão presentes déficits na compreensão da linguagem, porém estes costumam ser subestimados, já que as crianças podem se sair bem em usar o contexto para inferir sentido.

De acordo com Crestani et al. (2013), trata-se de um distúrbio que demanda conhecimento da aquisição e desenvolvimento da linguagem para ter diagnóstico preciso, no DEL o desempenho da linguagem das crianças não é compatível com sua capacidade intelectual não verbal. Assim, a identificação do DEL torna-se desafiadora.

Segundo Rapin e Allen (1988), as formas clínicas do DEL apresentam-se em alguns subtipos: (a) dispraxia verbal: déficit na programação motora da fala, compreensão da linguagem normal, fala não fluente ou ausente; (b) transtorno da programação fonológica: 
compreensão normal, fala fluente mas ininteligível; (c) misto-receptivo-expressivo: déficit sintático-fonológico: frases curtas e agramatismos, fluência e articulação alterada; (d) agnosia auditiva ou surdez verbal: compreensão verbal alterada, curtas ou produção de palavras, fluência e articulação alterada; (e) déficit semântico-pragmático: fala logorréica, compreensão deficiente, modos aberrantes de conversação; (f) déficit léxico-sintático: pseudogagueira, acesso lexical e construção sintática prejudicadas, compreensão de enunciados complexos deficiente.

\subsection{Critérios Diagnósticos para Distúrbio Específico de Linguagem (DEL) - Definição DSM-5}

A. Dificuldades persistentes na aquisição e no uso da linguagem em suas diversas modalidades (i.e., falada, escrita, linguagem de sinais ou outra) devido a déficits na compreensão ou na produção, inclusive:

i. Vocabulário reduzido (conhecimento e uso de palavras);

ii. Estrutura limitada de frases (capacidade de unir palavras e terminações de palavras de modo a formar frases, com base nas regras gramaticais e morfológicas).

iii. Prejuízos no discurso (capacidade de usar vocabulário e unir frases para explicar ou descrever um tópico ou uma série de eventos, ou ter uma conversa).

B. As capacidades linguísticas estão, de forma substancial e quantificável, abaixo do esperado para a idade, resultando em limitações funcionais na comunicação efetiva, na participação social, no sucesso acadêmico ou no desempenho profissional, individualmente ou em qualquer combinação.

C. O início dos sintomas ocorre precocemente no período do desenvolvimento.

D. As dificuldades não são atribuíveis a deficiência auditiva ou outro prejuízo sensorial, a disfunção motora ou a outra condição médica ou neurológica, não sendo mais bem explicadas por deficiência intelectual (transtorno do desenvolvimento intelectual) ou por atraso global do desenvolvimento

$\mathrm{Na}$ Classificação dos Transtornos Mentais e de Comportamento (CID-ı), o

Distúrbio Específico de Linguagem está classificado como Transtorno Específico do Desenvolvimento da Fala e Linguagem. Estes são transtornos nos quais os padrões normais 
de aquisição da linguagem estão perturbados desde os estágios precoces do desenvolvimento. As condições não estão diretamente atribuídas a anormalidades neurológicas ou do mecanismo da fala, a comprometimentos sensoriais, retardo mental ou fatores ambientais. A criança pode ser mais capaz de se comunicar ou entender em certas situações muito familiares do que em outras, mas a capacidade para linguagem em qualquer situação está comprometida.

Os transtornos específicos do desenvolvimento da fala e linguagem estão classificados em 6 categorias diferentes dentro do CID-ı: (I) Transtorno específico de articulação da fala; (2) Transtorno de linguagem expressiva; (3) Transtorno de linguagem receptiva; (4) Afasia adquirida com epilepsia (síndrome de Landau-Kleffner); (5) Outros transtornos do desenvolvimento da fala e linguagem; (6) Transtorno do desenvolvimento da fala e linguagem, não especificado.

\section{Diagnóstico diferencial entre Transtorno do Espectro Autista e Distúrbio Específico de Linguagem (DEL)}

Um dos grandes desafios encontrados com crianças com atraso na fala, ou dificuldades de interação com adultos ou outras crianças, é diferenciar entre vários possíveis diagnósticos.

De acordo com Faé et al. (2018), nos primeiros anos de vida, TEA e DEL se manifestam de forma parecida e sem muita especificidade, que pode estar relacionado ao nível de desenvolvimento cognitivo ou dificuldade com regulação emocional da criança, o que torna o diagnóstico diferencial entre ambos os transtornos mais complexo. Um importante fator confusional é que ambos apresentam atraso de fala no início da linguagem e/ou dificuldades de interação social.

Simms e Jin (2015) citam como exemplo que a reatividade emocional atípica ou evitação de olhar pode ser um sinal precoce de TEA, mas pode ser também devido à extrema timidez ou ansiedade, deficiências auditivas ou visuais, atrasos cognitivos ou distúrbios de comunicação não autistas. 
Faé et al. (2018) coloca que apesar de ambos os transtornos apresentarem características em comum, as crianças com TEA são mais debilitadas em sua funcionalidade quando comparadas as crianças com DEL, apresentando resultados inferiores em testes de habilidades cognitivas e de adaptação. Já a parte de linguagem, não apresenta diferença significativa entre os transtornos.

Segundo o DSM-5, os transtornos do espectro autista passam a ser avaliados a partir de dois domínios: comunicação social e interação social, padrões restritos e repetitivos de interesses, comportamentos ou atividades. Os transtornos de linguagem têm grande impacto na capacidade social do sujeito, mas há preservação do impulso de socialização. Estes se diferenciam do TEA por manifestarem reciprocidade e compartilharem interesses, emoções e afeto, capacidade de modificar o comportamento para adequar-se ao meio social (BAIRD; NORBURY, 2015).

De acordo com Faé et al. (2018), o TEA tem como principal característica além do prejuízo na comunicação verbal, déficits em vários outros aspectos da interação e alterações comportamentais distintas. As habilidades de linguagem são atrasadas e desordenadas tanto no TEA como no DEL e as principais distinções envolvem comportamentos sociais e imaginativos.

De acordo com Simms e Jin (2015), crianças com DEL possuem interesse em interações sociais e compartilham o que veem, fazem e encontram e são eficazes na comunicação não verbal, utilizando gestos e expressões faciais até adquirirem habilidades linguísticas apropriadas. Sua grande dificuldade na interação social é devido a sua capacidade linguística reduzida. Rotina é uma forma que crianças no DEL encontram para se organizarem e compreenderem seu contexto e como forma de transição de uma atividade para outra. Em contrapartida, crianças no TEA se beneficiam de comportamentos repetitivos e estereotipados para fins de autorregulação e/ou alívio de estresse.

Um importante fator no diagnóstico diferencial é a capacidade de imaginação e brincadeiras. Crianças com DEL geralmente gostam de imitar e histórias imaginativas, utilizam brinquedos de forma tradicional, as crianças com TEA demonstram pouco ou 
nenhum interesse em realizar jogos de imitação e costumam brincar de forma atípica em uma gama de possibilidades de objetos (FAÉ, 2018).

Segundo Simms e Jin (2015), um diagnóstico diferencial preciso deve iniciar com uma determinação do padrão cognitivo global da criança e habilidades de comunicação. $O$ ideal seria a avaliação formada por uma equipe multidisciplinar que pode ser composta por psicólogo infantil, fonoaudiólogo, pediatra do desenvolvimento, psiquiatra infantil e especialista em educação. Porém, os níveis funcionais e de desenvolvimento de uma criança podem ser avaliados em um ambiente pediátrico por vários meios como uma anamnese cuidadosa de pais e/ou cuidadores focando especificamente na fala e linguagem da criança, habilidades motoras. Questionários e formulários de avaliação feitos por adultos com familiaridade com a criança podem ser de grande auxílio com informações precisas. Existem poucos sintomas individuais exclusivos de qualquer transtorno. Faz-se necessário que o sintoma da criança seja considerado no contexto de seu perfil cognitivo e habilidades verbais.

\section{PERFIL NEUROPSICOLÓGICO DO DEL E TEA}

\section{I Perfil neuropsicológico do Distúrbio Específico de Linguagem (DEL)}

A definição de DEL é uma alteração de linguagem primária que ocorre na ausência de perda auditiva, alteração no desenvolvimento cognitivo e desenvolvimento motor da fala, síndromes, perturbações globais do desenvolvimento, alterações neurossensoriais e lesões neurológicas adquiridas, e que impede o desenvolvimento da linguagem de forma esperada (Fresneda e Mendonza, 2005). No entanto, não há disfunção geral no DEL, mas alguns domínios podem revelar prejuízos.

Entre esses prejuízos, os relacionados a memória são apontados como bons marcadores de DEL e alterações de memória verbal são apresentados como um dos principais déficits deste quadro. Ainda que os portadores de DEL apresentem boa capacidade não verbal, existem prejuízos encontrados em alguns estudos relacionados a tarefas de memória não visual, é preciso considerar esse fator, pois sugere que não se beneficiam de pistas visuais para auxílio no desenvolvimento de aspectos não verbais. Estudos realizados no campo da memória verbal, concluem que quando comparadas indivíduos normais, o 
grupo com DEL apresenta resultados inferiores. A seguir será apresentado um estudo realizado em Coimbra, realizado por Coelho et al. 2016 que compara prejuízos cognitivos de indivíduos com e sem DEL. Através do teste Memória de História, que avalia capacidade de aprendizagem, retenção, evocação e reconhecimento de material auditivo e verbal, A Figura Complexa de Rey, que avalia processos visuoespaciais e construtivos, memória visual e funções executivas, foi medida a capacidade de memória dos grupos.

Segundo Coelho et al. 2013, quando avaliado o nível de atenção, os estudos realizados não puderam ser conclusivos. É possível que os indivíduos com DEL apresentem em determinados tipos de atenção, resultados semelhantes aos indivíduos normais, enquanto apresentam maiores dificuldades em atenção seletiva e auditiva, por exemplo. O teste usado para avaliar a atenção no estudo de Coelho et al. 2013 foi Trail Making Test, que avalia a atenção seletiva, atenção dividida e velocidade motora e o Teste de Barreiragem de 2 e 3 sinais, que mede atenção seletiva e atenção sustentada.

Em relação às funções executivas, foi concluído que existem déficits principalmente na inibição de respostas, organização e das estratégias de planejamento com vista a solução de problemas. Para avaliar este aspecto, além da Figura de Rey foi utilizada a Torre de Coimbra que avalia funções executivas, nomeação e capacidade de planejamento, monitorização, autorregulação e resolução de problemas.

Para avaliar a linguagem, os testes escolhidos foram Fluência Verbal Semântica e Fonêmica, que avaliam aptidão para gerar palavras de acordo com duas categorias: fonêmica e semântica, e os testes de Nomeação Rápida de Formas e Cores e de Números que avaliam a aptidão para aceder e produzir palavras familiares de maneira rápida.

Em relação a resultados, nos testes de memória, foram identificados importantes diferenças entre os grupos na parte de evocação e reconhecimento imediato e evocação e reconhecimento diferido. Nos testes de atenção e funções executivas, o grupo com DEL apresentou resultados mais baixos em relação ao outro grupo.

Nos testes que avaliaram atenção e funções executivas, como o Trail Making, os indivíduos com DEL diferenciaram-se significativamente do outro grupo no número de erros cometidos, mas não na velocidade, ainda que apresentassem resultados inferiores. No 
teste Torre de Coimbra, o grupo com DEL apresentou resultados mais baixos no número de problemas resolvidos e no tempo de planejamento. Houve diferenças significativas também, no total de erros.

Os resultados de linguagem, no teste de Nomeação rápida o grupo com DEL apresentou resultados inferiores tanto no número de erros, quanto no tempo. Em relação à Fluência Verbal, os resultados foram mais baixos em Fluência Verbal Semântica, na Fluência Fonêmica, as diferenças não foram significativas.

$\mathrm{Na}$ avaliação das funções visuoperceptivas, o grupo com DEL obteve resultados na Figura de Rey significativamente mais baixos do que o grupo em comparação. O grupo com DEL manifesta erros de convergência e perseverações.

Em geral, os testes marcam déficits importantes de funcionamento neuropsicológico nas crianças com DEL em comparação ao outro grupo, principalmente em na memória visual, memória de histórias, onde obtiveram resultados inferiores apontando dificuldade no processo de evocação e codificação visual. Sugere-se também, que os indivíduos com DEL possuam dificuldades de operar estímulos visuais, complexos ou simples como observados na Figura de Rey.

No teste de memória verbal, houve diferenças significativas. As grandes dificuldades do grupo com DEL aparecem nos processos de codificação da informação verbal, assim como mostram os resultados inferiores de evocação imediata em Memória de Histórias. Foram identificadas dificuldades tanto na evocação quanto no reconhecimento e constata-se adicionalmente que essas dificuldades podem se localizar na codificação da informação, que uma vez não codificada, é mais difícil lembrar independente das condições em que foi solicitada. Assim, os déficits nos processos de codificação dos indivíduos com DEL, podem estar relacionados a memória de trabalho verbal e processamento de informação auditiva. Contudo, se a evocação e reconhecimento estão prejudicados, pode haver implicação de desatenção, dificuldades de compreensão, ou mesmo falta de interesse pela tarefa.

À nível de atenção, constatou-se que o grupo com DEL apresenta limitações na capacidade de atenção sustentada e dividida. Eles apresentaram ritmo mais lento na execução das tarefas e no processamento da informação. No entanto, há de se levar em 
consideração que quando as exigências das tarefas são reduzidas, o grupo com DEL possui recursos suficientes ao nível de atenção para um bom desempenho, o que não aconteceria caso as exigências fossem elevadas como no Teste Barragem de 2 Sinais.

A avaliação das funções executivas também se mostrou prejudicada, com resultados inferiores na capacidade de flexibilidade cognitiva, planificação, resolução de problemas e inibição de condutas como demonstra-se no Teste Torre de Coimbra. A maior parte dos erros acontece pela falta de planejamento, relacionados a dificuldade de monitorização de desempenho, processar informações simultâneas e manter regras na memória de trabalho.

Foram encontrados déficits em linguagem na parte de nomeação rápida de números e de formas e cores, na fluência verbal semântica e fluência verbal total. Os estudos mostram que indivíduos com DEL tem dificuldades na evocação de palavras com tendência a lentidão e cometem mais erros (Lahey E Edwards, 1999). A menor evocação de palavras pode ser atribuída a dificuldades de acessar o sistema mnésico, a um problema de ritmo da produção, dificuldades de aplicação de estratégias eficazes, ao esquecimento das instruções iniciais, devido a memória de trabalho prejudicada ou mesmo incapacidade de monitorar suas respostas.

\subsection{Perfil Neuropsicológico do Transtorno do Espectro Autista (TEA)}

Segundo o DSM-5, o Transtorno do Espectro Autista (TEA) é classificado como um transtorno do desenvolvimento que tem prejuízos em duas esferas principais, o social (comunicação e interação social) e não social (padrões restritos e repetitivos de comportamentos de interesses e atividades).

De acordo com Silva e Mulick (2009), a avaliação de diagnóstico precisa ser feita por equipe multidisciplinar. Existem diversos instrumentos que podem auxiliar na identificação de sintomas de autismo durante o próprio processo de avaliação. Um dos instrumentos mais utilizados é o CARS - Childhood Autism Rating Scale (Escala de Avaliação de Autismo na Infância), onde a severidade dos sintomas e os comportamentos da criança são avaliados de acordo com informações das entrevistas com pais e cuidadores da criança. Essa escala compreende o comportamento em I4 domínios que geralmente são afetados no autismo. 
Esses itens incluem: relações pessoais, imitação, resposta emocional, comunicação verbal e não verbal, entre outros.

Outros instrumentos utilizados são o ADI-R - Autism Diagnostic Interview Revised (Entrevista Diagnóstica para Autismo), que segundo Silva e Mulick (2009), correspondem a uma entrevista semiestruturada com os pais e o ADOS - Autism Diagnostic Observation Schedule (Observação Diagnostica Programática para Autismo) que é um programa semiestruturado de atividades e entrevistas com a criança, um processo diagnóstico que fornece informações acerca do funcionamento cognitivo e adaptativo da criança, que é fundamental para a formação de um plano de intervenção.

Neumann et al. (2016) aponta que o subteste Cubos das escalas Weschler que reúnem classificação de imagens por serie, os indivíduos com TEA mostram falta de tendência natural para unir partes de informações para formar um todo provido de significado (coerência central), sendo este um marcador importante do autismo.

De acordo com um estudo de Ventola et al. (2006), comparando crianças dentro do TEA e crianças portadoras de outros transtornos de atraso de desenvolvimento, as crianças com TEA foram mais prejudicadas em atenção compartilhada, habilidade de imitação, resposta empática, apontar para manifestar interesse, interesse em outras crianças.

Para verificação de comportamentos de atenção compartilhada, um instrumento usado é o CHAT, para identificação de autismo aos I8 meses de idade. Segundo Oosterling et al. (2009) o CHAT avalia não só comportamentos de atenção compartilhada como brincadeiras de faz-de-conta utilizando um relatório parental e observação de profissionais de saúde com testes diretos.

Segundo um estudo de Fernandes et al. (2018) com crianças dentro do TEA e crianças com deficiência intelectual, foi constatado que o grupo no TEA apresentava maior dificuldade em tarefas verbais, propondo que que o fator verbal influencie o desempenho. Em relação a funções executivas, foram identificados prejuízos em planejamento, processamento de memória de trabalho, controle inibitório e flexibilidade cognitiva nos casos do grupo de TEA. Contudo, este impacto não se apresenta da mesma forma em todos os pacientes dentro do TEA. 
Faé et al. (2018) sinaliza que o TEA apresenta não só prejuízo na comunicação verbal, mas também déficits em aspectos de interação social e alterações comportamentais. Os portadores de TEA apresentam debilidade funcional, demonstram empatia limitada.

\section{CONSIDERAÇÕES FINAIS}

A proposta do presente trabalho foi estudar a partir da literatura, estudos e pesquisas disponíveis as principais diferenças entre os perfis dos portadores do Transtorno do Espectro Autista e o Distúrbio Específico de Linguagem.

Segundo a revisão realizada, os portadores de TEA apresentam prejuízos importantes na comunicação verbal e déficits na relação social. Estão presentes em ambos os transtornos, prejuízos e atrasos na linguagem e um importante fator de distinção entre eles são comportamentos sociais e imaginativos.

Os portadores de DEL procuram interagir com o meio social e conseguem se comunicar de maneira não verbal, sua dificuldade aparece em geral como consequência da limitada capacidade verbal.

Indivíduos dentro do TEA apresentam falta de interesse por outras pessoas e pelo meio social além de alterações comportamentais como comportamentos estereotipados e repetitivos, sintomas que não estão presentes no DEL.

Outro importante fator a ser considerado no diagnóstico diferencial é a capacidade imaginativa. Os portadores de DEL de modo geral gostam de jogos de imitação e contar histórias de faz-de-conta, utilizam os brinquedos da maneira padrão. Em contrapartida os portadores de TEA demonstram pouco ou nenhum interesse em imitação e utilizam brinquedos de maneiras atípicas. O que diferencia um do outro é o foco e a intensidade destes comportamentos.

Portadores de DEL se beneficiam de rotina para organização e compreensão de seu contexto ambiental, e os TEA entram em comportamentos repetitivos para autoestimulação ou alívio de estresse. Conclui-se que o maior fator distintivo entre os transtornos envolve comportamentos imaginativos. 


\section{REFERÊNCIAS BIBLIOGRÁFICAS}

AMERICAN PSYCHIATRIC ASSOCIATION. DSM-IV. Manual Diagnóstico e Estatístico de Transtornos Mentais. Porto Alegre: ARTMED, 4a. ed., 2002.

ANDRADE, M.J. et al. Desempenho de escolares em testes de atenção e funções executivas: estudo comparativo. 2016.

BAIRD, G; NORBURY, C.F. Social (pragmatic) communication disorders and autism spectrum disorder. 2015 .

COELHO, Sandra; AlBUQUERQUE, Cristina Petrucci; SIMOES, Mário Rodrigues. Distúrbio Específico de Linguagem: Caracterização Neuropsicológica. Paidéia (Ribeirão Preto), v. 23, n. 54, p. 31-41, 2013.

CRESTANI, Anelise Henrich et al. Distúrbio específico de linguagem: a relevância do diagnóstico inicial. Rev. CEFAC, São Paulo, v. 15, n. I, p. 228-237, 2013.

FAÉ, I.G. et al. Diagnóstico diferencial entre transtornos de espectro autista e transtorno específico de linguagem receptivo e expressivo: uma revisão integrativa. 2018.

FERNANDES, S.C. et al. Evidências de diagnóstico diferencial entre Transtorno do 1482 Espectro Autista (TEA) e Transtorno do desenvolvimento intelectual (TDI): análise de casos. 2018.

FRESNEDA, M.D, MENDOZA, E. Trastorno específico del lenguaje: concepto, clasificaciones y criterios de identificación. Rev Neurol. 2005.

JUliO-COSTA, A., ANTUNES, A. M. Transtorno do Espectro Autista na Prática Clínica. São Paulo: Pearson Clinical Brasil, 2017.

LAHEY, M., EDWARDS, J. Naming erros of children with specific language impairment. J Speech Lang Hear Res. 42 (I): 195-205, 1999.

KANNER, L. Autistic Distubances of affective contact. New Child, v. 2, p. 217, 1943.

NEUMANN, D.M. et al. Avaliação neuropsicológica do transtorno do espectro autista. 2016.

OOSTERLING, I.J.et al. Comparative Analysis of Three Screening Instruments for Autism Spectrum Disorder in Toddlers at High Risk. J Autism Dev Disord, 2009.

SILVA, M.; MULICK, A. J. Diagnosticando o transtorno autista: aspectos fundamentais e considerações práticas. Psicologia Ciência e Profissão. Brasília, v. 29, n. I, p. II6-I3I, 2009. 
SIMMS, M.D.; JIN, M.J. Autism, Language disorder and social (pragmatic) communication disorder: DSM-V and differential diagnoses. Pediatrics in Review. August 2015 .

VENTOLA, P. et al. Differentiating between Autism Spectrum Disorders and Other Developmental Disabilities in Children Who Failed a Screening Instrument for ASD. J Autism Dev Disord, 2007. 